\title{
Review
}

Xavier Filella*, María Dolores Albaladejo, Juan Antonio Allué, Miguel Angel Castaño, Daniel Morell-Garcia, Maria Àngels Ruiz, María Santamaría, María José Torrejón and Nuria Giménez

\section{Prostate cancer screening: guidelines review and laboratory issues}

https://doi.org/10.1515/cclm-2018-1252

Received November 22, 2018; accepted April 21, 2019; previously published online May 23, 2019

\section{Abstract}

Background: Prostate-specific antigen (PSA) remains as the most used biomarker in the detection of early prostate cancer (PCa). Clinical practice guidelines (CPGs) are produced to facilitate incorporation of evidence into clinical practice. This is particularly useful when PCa screening remains controversial and guidelines diverge among different medical institutions, although opportunistic screening is not recommended.

Methods: We performed a systematic review of guidelines about PCa screening using PSA. Guidelines published since 2008 were included in this study. The most updated version of these CPGs was used for the evaluation.

\footnotetext{
*Corresponding author: Xavier Filella, Evidence Based Laboratory Medicine Commission and Biological Markers of Cancer Commission, Spanish Society of Laboratory Medicine (SEQC-ML), Barcelona, Spain; and Department of Biochemistry and Molecular Genetics (CDB), Hospital Clinic, IDIBAPS, Barcelona, Spain, E-mail:xfilella@clinic.cat

María Dolores Albaladejo: Evidence Based Laboratory Medicine Commission, Spanish Society of Laboratory Medicine (SEQC-ML), Barcelona, Spain; and Department of Clinical Analysis and Biochemistry, Hospital General Universitario Santa Lucía, Cartagena, Spain

Juan Antonio Allué: Evidence Based Laboratory Medicine Commission, Spanish Society of Laboratory Medicine (SEQC-ML), Barcelona, Spain; and Synlab Diagnosticos Globales, Sevilla, Spain Miguel Angel Castaño: Evidence Based Laboratory Medicine Commission, Spanish Society of Laboratory Medicine (SEQC-ML), Barcelona, Spain; and Department of Biochemistry, Hospital Clínico Universitario Juan Ramón Jiménez, Huelva, Spain Daniel Morell-Garcia: Evidence Based Laboratory Medicine Commission, Spanish Society of Laboratory Medicine (SEQC-ML), Barcelona, Spain; and Department of Laboratory Medicine, Hospital Universitari Son Espases, Palma de Mallorca, Spain
}

Results: Twenty-two guidelines were selected for review. In 59\% of these guidelines, recommendations were graded according to level of evidence $(n=13)$, but only $18 \%$ of the guidelines provided clear algorithms $(n=4)$. Each CPG was assessed using a checklist of laboratory issues, including pre-analytical, analytical, and post-analytical factors. We found that laboratory medicine specialists participate in $9 \%$ of the guidelines reviewed $(n=2)$ and laboratory issues were frequently omitted. We remarked that information concerning the consequences of World Health Organization (WHO) standard in PSA testing was considered by only two of 22 CPGs evaluated in this study. Conclusions: We concluded that the quality of PCa early detection guidelines could be improved properly considering the laboratory issues in their development.

Keywords: clinical practice guidelines; prostate cancer; prostate-specific antigen (PSA); screening.

Maria Àngels Ruiz: Evidence Based Laboratory Medicine Commission, Spanish Society of Laboratory Medicine (SEQC$\mathrm{ML}$ ), Barcelona, Spain; and Department of Laboratory Medicine, Fundació Hospital de l'Esperit Sant, Santa Coloma de Gramenet, Barcelona, Spain

María Santamaría: Evidence Based Laboratory Medicine Commission, Spanish Society of Laboratory Medicine (SEQC-ML), Barcelona, Spain; and Department of Biochemistry, Hospital Clínico Universitario Lozano Blesa, Zaragoza, Spain

María José Torrejón: Evidence Based Laboratory Medicine Commission, Spanish Society of Laboratory Medicine (SEQC-ML), Barcelona, Spain; and UGC of Clinical Analysis, Hospital Clínico San Carlos, Madrid, Spain

Nuria Giménez: Evidence Based Laboratory Medicine Commission, Spanish Society of Laboratory Medicine (SEQC-ML), Barcelona, Spain; Committee of Evidence-Based Laboratory Medicine (C-EBLM), International Federation of Clinical Chemistry and Laboratory Medicine (IFCC), Milano, Italy; Research Unit, Research Foundation Mútua Terrassa, Universitat de Barcelona, Barcelona, Spain; and Laboratory of Toxicology, Universitat Autònoma de Barcelona, Barcelona, Spain 


\section{Introduction}

Prostate cancer (PCa) is the most prevalent cancer in males in Western countries. In Europe, an incidence of 450,000 new cases and a mortality of 107,000 cancer deaths per year have been estimated for 2018 [1]. Prostate-specific antigen (PSA) remains as the most used biomarker in the detection of early PCa. However, since 2009, PSA usefulness in PCa screening has been widely argued following the opposite results obtained in the two largest randomized screening studies. The European Randomized Study of Screening for Prostate Cancer (ERSPC), enrolling 162,387 men between 50 and 74 years old from seven European countries, found a PCa-specific mortality reduction of $20 \%$ in the screened group after a median follow-up of 9 years [2]. In evident contrast, the Prostate, Lung, Colorectal, and Ovarian (PLCO) Screening Trial, based on 76,693 men, found no differences in the PCa-specific mortality after 7 years of follow-up between the screened group and the control group [3]. Furthermore, these opposite results were confirmed by both groups increasing the time of follow-up and differences were confirmed by the ERSPC trial [4].

In 2011 the United States Preventive Services Task Force (USPSTF) strongly advise against PSA screening based on a review of six well-done trials, underlying harms related to subsequent evaluation and treatments [5]. Nevertheless, several studies showed the evidence that screening reduces the risk of metastasis both at diagnosis and during follow-up [6, 7]. Furthermore, Gulati et al. [8] suggested that discontinuing PSA screening for all men may generate many avoidable cancer deaths in the next years. On the other hand, Stephan et al. [9] remarked on methodological limitations in the meta-analysis showing no evidence of a PCa-specific mortality reduction, suggesting the value of multivariable risk-prediction tools to select appropriate treatment or active surveillance. Additionally, in a recent review, Carlsson and Roobol [10] underlined data emerging in last years that suggest a new approach to PCa screening according to PSA-based risk stratification at an early age. Similarly, Eapen et al. [11] postulated in favor of a smarter screening approach, based on relatively infrequent PSA testing, consistent use of multivariable risk stratification, and selective treatment focused on patients with high grade $\mathrm{PCa}$.

Clinical practice guidelines (CPGs) are produced to facilitate incorporation of evidence into clinical practice. This is particularly useful when PCa screening remains controversial and guidelines diverge among different medical institutions. Ideally CPGs from different organizations should be based on high quality methodology to achieve similar clinical recommendations. Unfortunately, despite of the enormous energies invested in its realization, the value of CPGs varies considerably. Clinicians should consider recommendations of varying evidence levels differently. A multidisciplinary approach, the description of the sources of information used, and recommendations graded according to level of evidence are necessary items to be considered in a high-quality CPG.

The evidence-based laboratory medicine commission of the Spanish Society of Laboratory Medicine (SEQC-ML) has observed that, despite the obvious involvement of the clinical laboratory in the PCa screening using PSA, some technical aspects have been unremarked among clinicians [12].

We critically review the characteristics of guidelines about PCa detection, focusing the attention on the laboratory issues considered. In addition, we propose a checklist including several laboratory issues related to PSA measurement that in our opinion should be considered during the development of clinical practice guidelines.

\section{Materials and methods}

\section{Information sources, search strategy and study selection}

A systematic search was undertaken using several electronic databases: Medline/PubMed, Web of Science and Scopus. A strategic search was done by one reviewer looking for combinations of the following search terms: as publication type ("Guideline", "Practice Guideline"), as medical subject heading MeSH ("Guidelines as Topic", "Prostatic Neoplasms", "Prostate-Specific Antigen", "Mass Screening", "Early Detection of Cancer", "Diagnosis") and as free search terms ("guideline", "prostate cancer”, "prostate specific antigen”, "screening”, "diagnosis"). Guidelines published since 2008 were included in this study. The most updated version of these CPGs was used for the evaluation. This scientific literature search was complemented with three international guideline websites: National Guidelines Clearinghouse [13], Guidelines International Network Web site [14] and National Institute for Health and Care Excellence (NICE) [15].

\section{Eligibility criteria}

All the guidelines were considered eligible for inclusion if they met the following criteria: publication type guideline, published in the last 10 years, written in English language. 


\section{Data collection process}

Two of the authors participated in the final process of guideline selection. The main information, overall recommendation and remarkable quality information of each guideline was extracted by two researcher and independently checked for accuracy by the other researchers. Disagreements between researchers were resolved by discussion.

Each CPG was assessed using a checklist of laboratory issues following the criteria described by Aakre et al. [16]. These criteria were adapted for the PSA assay. Previous versions of the selected GPCs were also checked for this purpose.

The Appraisal of Guidelines Research and Evaluation II (AGREE II) instrument was used to assess the quality of guidelines. This tool has 23 items distributed in six domains: scope and purpose (1), stakeholder involvement (2), rigor of development (3), clarity of presentation (4), applicability (5) and editorial independence (6). Each guideline was rated by four independent appraisers trained using the online training tools recommended by the AGREE collaboration. We also followed the AGREE II instrument guideline to calculate all the scores.

\section{Results}

The study selection process is summarized in Figure 1.

A summary of the most relevant characteristics of the 22 selected PCa guidelines are shown in Table 1 [1738], including information concerning, among others, overall recommendation, laboratory issues considered, the description of the sources of information used, and recommendations graded according to the level of evidence. Shared decision considering benefits and harms of PCa screening is a frequent criterion in the evaluated guidelines, while mass screening is not recommended in any guideline. Differences among guidelines go from the non-restricted criteria counseled by the Canadian Urological Association to the recommendation against screening counseled by the American College of Physicians or the Australian Government National Health and Medical Research Council. Furthermore, in the majority of CPGs, screening is explicitly not recommended for men higher than 70 years old or with a life expectancy $<10$ years.

Table 2 shows a list of laboratory issues that should be examined for PSA measurement. CPGs should report information about these laboratory requirements for high quality results. The list was adapted from Aakre et al. [16]

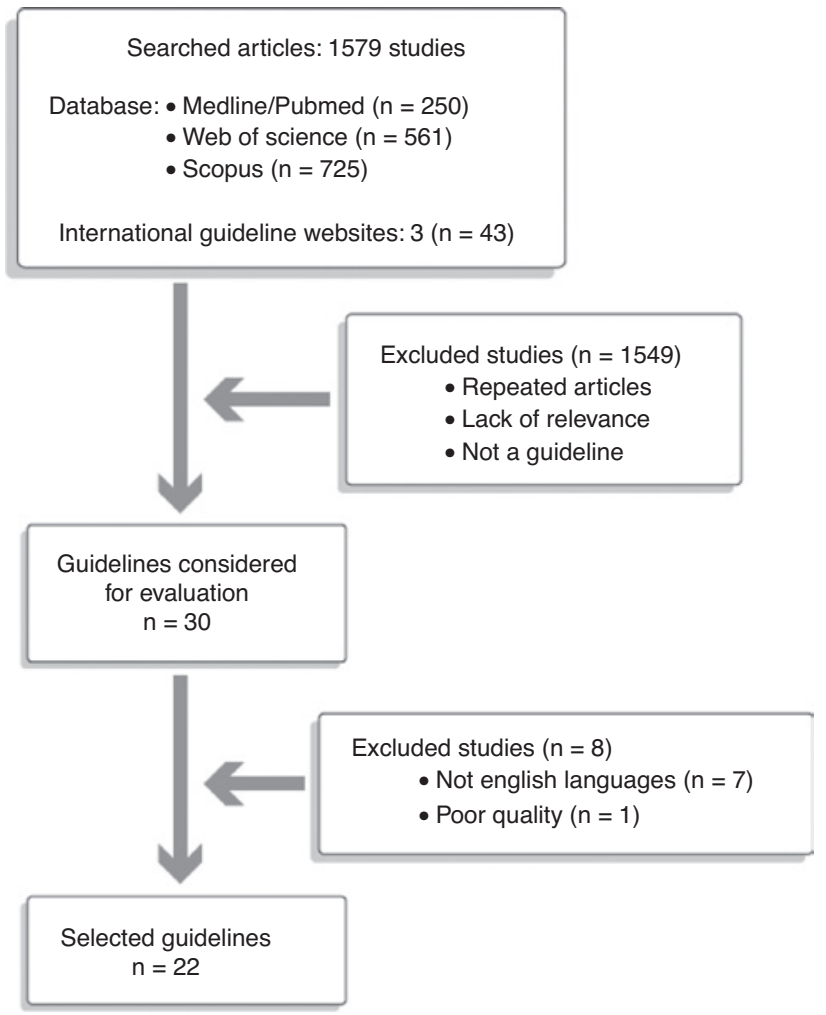

Figure 1: Study selection process.

but modified according to the specific criteria for PSA [39]. The issues were classified following three categories: pre-analytical, analytical and post-analytical phases. Specifically, guidelines should report information about the standard reference material used in the measurement of PSA and how reference ranges were established.

We found data concerning consequences of the adoption of the standard reference material from the World Health Organization (WHO) in PSA testing in only two of 22 CPGs evaluated in this study, the National Academy of Clinical Biochemistry (NACB) and the National Comprehensive Cancer Network (NCCN) guidelines. Also, the guideline of the Canadian Urological Association informs of $20 \%-25 \%$ differences in the concentration of PSA among laboratories.

Previous versions of the selected GPCs were also checked for this purpose. We found relevant data in previous versions of American Urological Association (AUA) guidelines, corresponding to 2009, and the European Association of Urology (EAU) guidelines, corresponding to 2013 and 2015, respectively.

Three of the six CPGs considering laboratory issues remark that assays using the WHO standard offer results 20\%-25\% lower than those using the initial Hybritech (C) standard. The other three guidelines only refer that results 


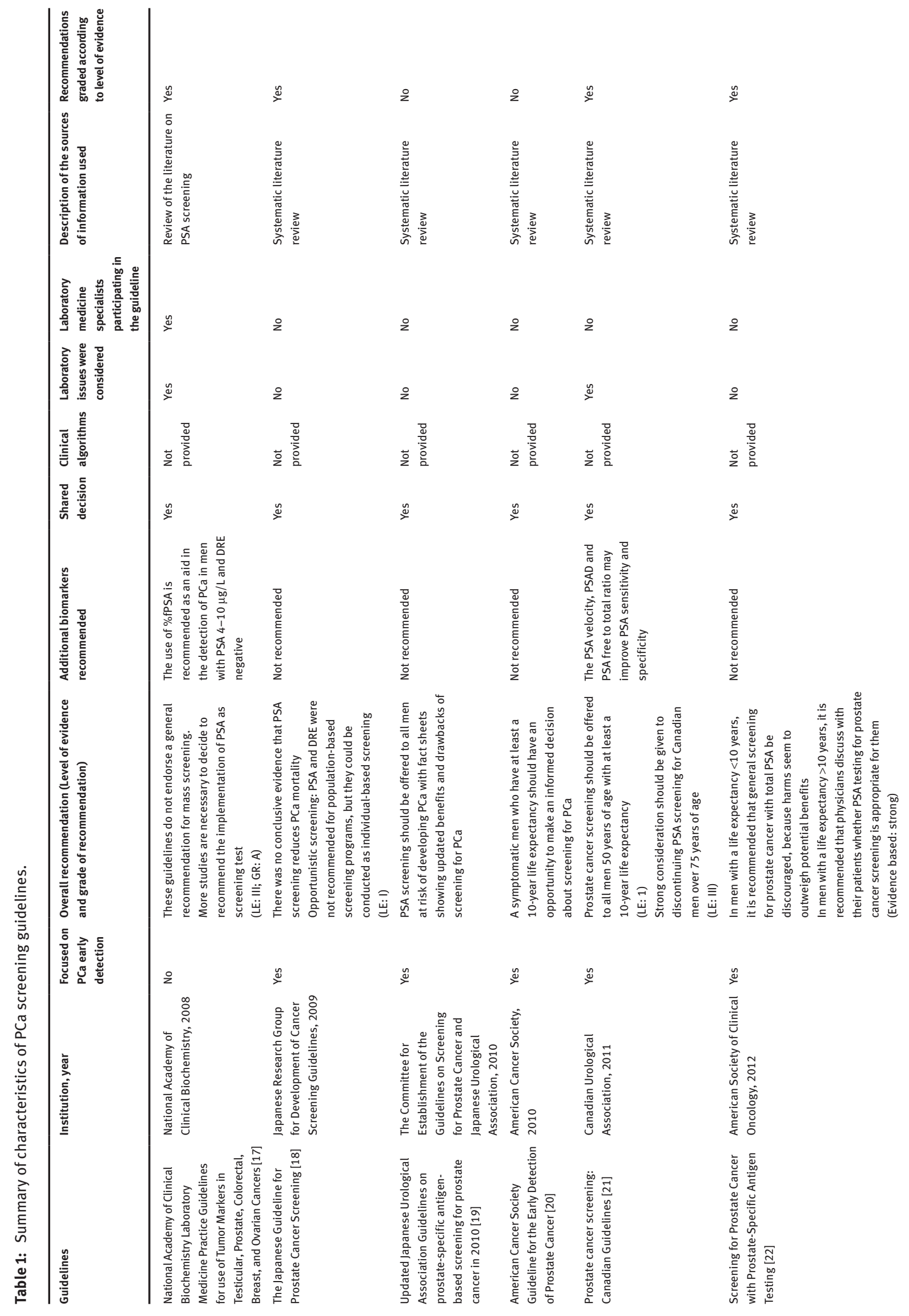




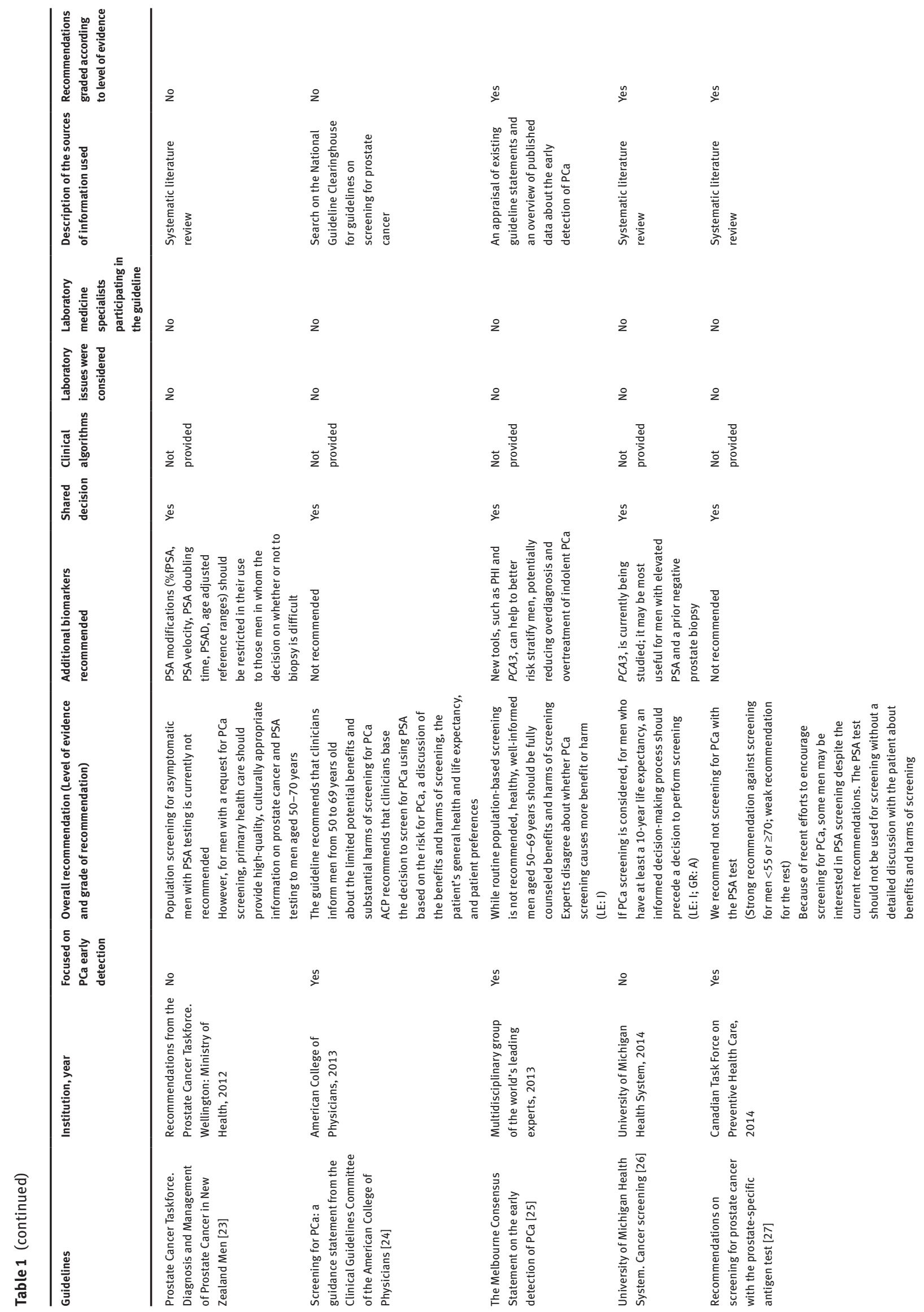




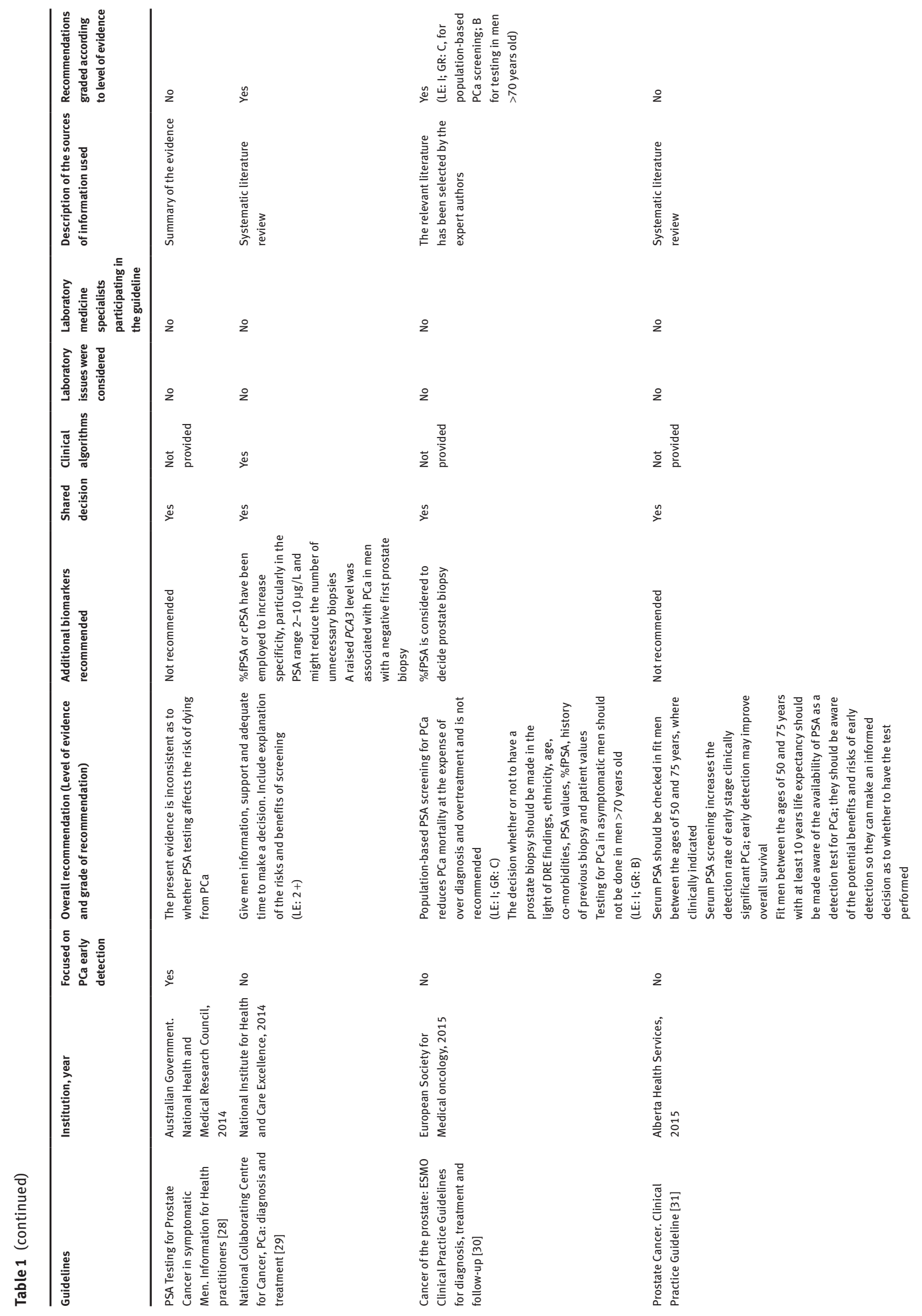




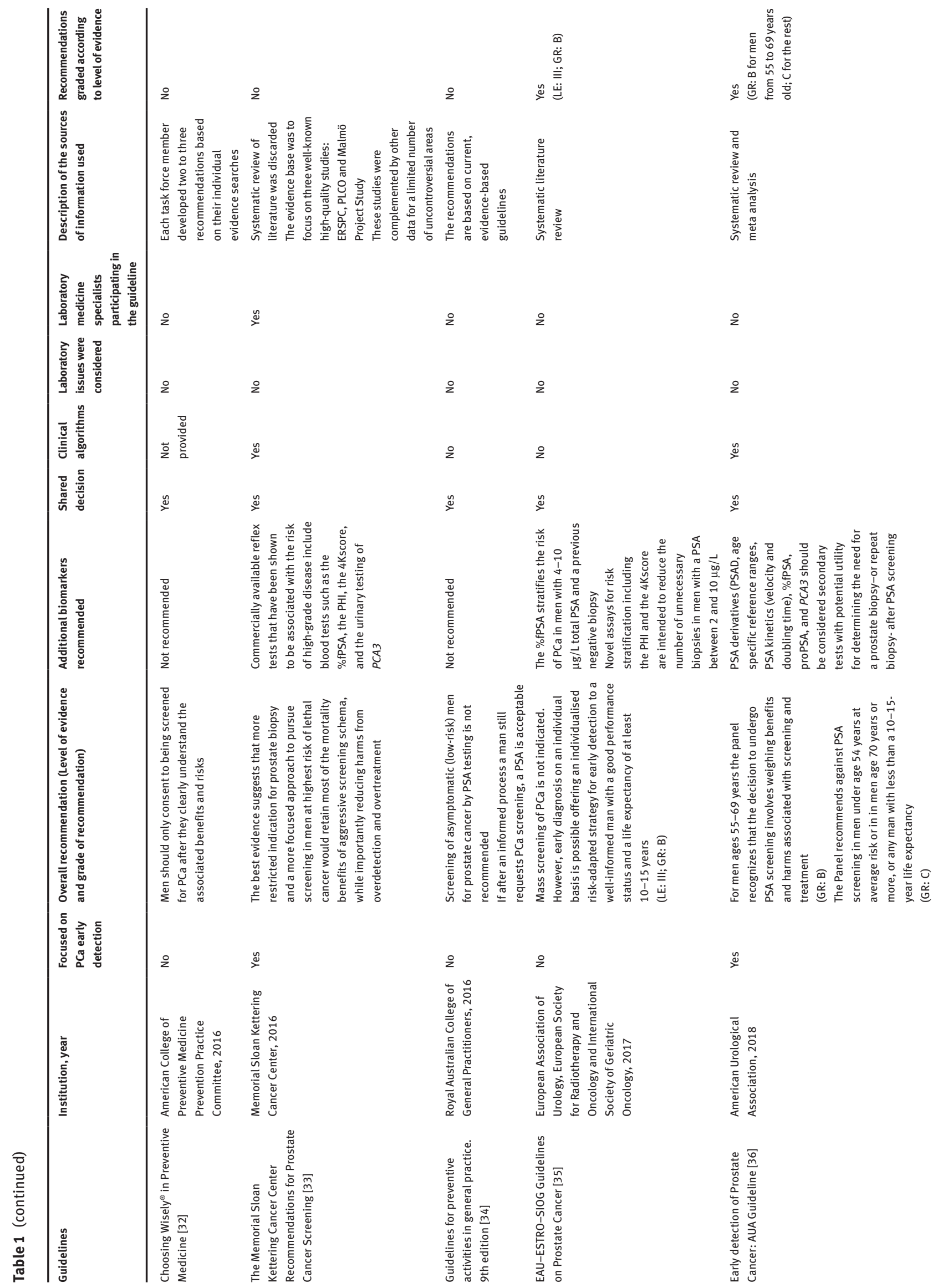




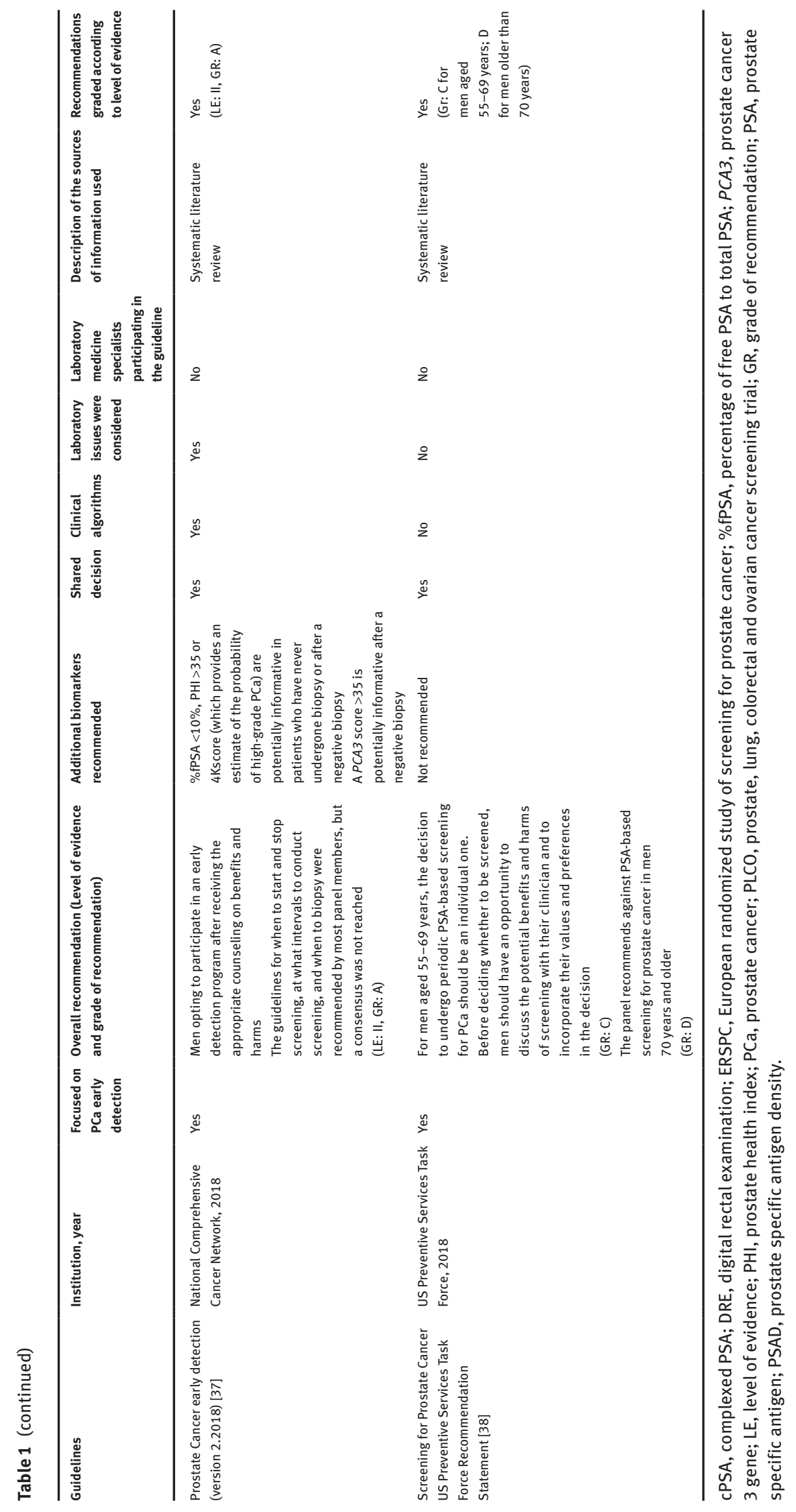


Table 2: List of laboratory issues that should be considered for PSA assays in a clinical practice guideline.

\begin{tabular}{lll}
\hline Pre-analytical phase & Analytical phase & $\begin{array}{c}\text { Post-analytical } \\
\text { phase }\end{array}$ \\
\hline Sampling handling: sample type; stability of specimen on & $\begin{array}{l}\text { Methodology: information about the assay used in the } \\
\text { measurement of PSA; standard used in the calibration } \\
\text { storage; maximum number of freezing/thawing cycles }\end{array}$ & $\begin{array}{l}\text { Assay specific } \\
\text { reference range }\end{array}$ \\
$\begin{array}{l}\text { Prostate manipulation: digital rectal examination; prostate } \\
\text { massage; cystoscopy; transrectal ultrasound; prostate }\end{array}$ & $\begin{array}{l}\text { Quality issues: imprecision intra- and inter-assay; } \\
\text { external quality assessment; accreditation of the }\end{array}$ \\
biopsy; bladder catheterization & $\begin{array}{l}\text { laboratory } \\
\text { Sexual activity (ejaculation) }\end{array}$ & $\begin{array}{l}\text { Analytical interferences caused by lipemia, hemolysis } \\
\text { or bilirubin } \\
\text { Interferences caused by medication (5-alfa-reductase }\end{array}$ \\
inhibitors) & $\begin{array}{l}\text { Interference from heterophilic or human anti-mouse } \\
\text { antibodies (HAMA) }\end{array}$ \\
Intraindividual variability &
\end{tabular}

between different PSA assays are not interchangeable. A summary of these characteristics is shown in Table 3 [17, 37, 45, 48, 50]. The references cited in these guidelines about PSA standardization are also indicated.

We used the AGREE II instrument to evaluate the quality of the guidelines (Table 4). The overall assessment score ranged from $39.5 \%$ to $97 \%$ and 13 (59\%) of the 22 guidelines obtained an overall assessment score higher to $70 \%$. The highest score was reached by the NICE, the Canadian Task Force on Preventive Health Care and the AUA guidelines.

\section{Discussion}

The usefulness of PSA as a screening test for reducing PCa mortality has been an area of intense controversy. Nowadays, PCa guidelines recommend against routine PSA testing or emphasize that the decision to undergo early PSA testing should be a shared decision between the patient and his physician, considering potential benefits and harms. Nonetheless, discussion about the opportunity of screening remains. Actually, a balance between benefits and harms is the key point to decide to screen. So, active surveillance has been proposed to mitigate harms related with overtreatment in patients with indolent PCa. On the other hand, recently, several studies have noted a reduction in PSA screening rates following the USPSTF [5, 52] recommendation against systematic PSA screening in general population [53-55]. Fleshner et al. [56] have recently remarked that the withdrawal of PSA screening would prevent all cases of overdiagnosis, but would fail to prevent $100 \%$ of avoidable deaths, leading to a $13 \%-20 \%$ increase in PCa-related deaths. These data demonstrate that harms associated with no screening must be also considered and suggest that the process of reviewing evidences and updating recommendations must be continuous. This is reflected in the remarkable changes in the recommendations of the US Preventive Services Task Force along the last decade. In 2008, the panel indicated for men younger than 75 years old that current evidence was insufficient to assess the balance of benefits and harms of PCa screening [57]. Later on, in 2012, the group recommended against PCa screening [52], but, more recently, in 2018, the panel recommends a shared decision considering benefits and harms for men aged from 55 to 69 years [38].

On the other hand, additional reflex tests in blood (Prostate Health Index, 4Kscore) or urine (PCA3) has been proposed by several guidelines [25, 26, 29, 33, 35-37] to increase PSA specificity and potentially to decrease the overdiagnosis of indolent PCa. Furthermore, recent data have demonstrated that these biomarkers can also improve the cost-effectiveness of PCa screening [58, 59], showing that is more cost-effective the use and development of screening tests than the use of tests after a negative biopsy [60].

The quality of PCa CPGs is heterogeneous according to published data. Heterogeneity of results has been emphasized by Gupta et al. [61] using the AGREE II instrument to assess the quality of 13 CPGs selected from 1999 to 2014. The authors showed that the guidelines from the NICE and AUA had the best scores in most domains using this tool. We share the same conclusion, remarking the heterogeneity of quality in guidelines and having obtained a high overall score for NICE and AUA guidelines, as well. In contrast, Qaseem et al. [24] gave a low overall quality rate to the AUA guideline, remarking the high quality of the American Cancer Society (ACS) guideline. All these results show the need for improving CPGs methodology and quality. 


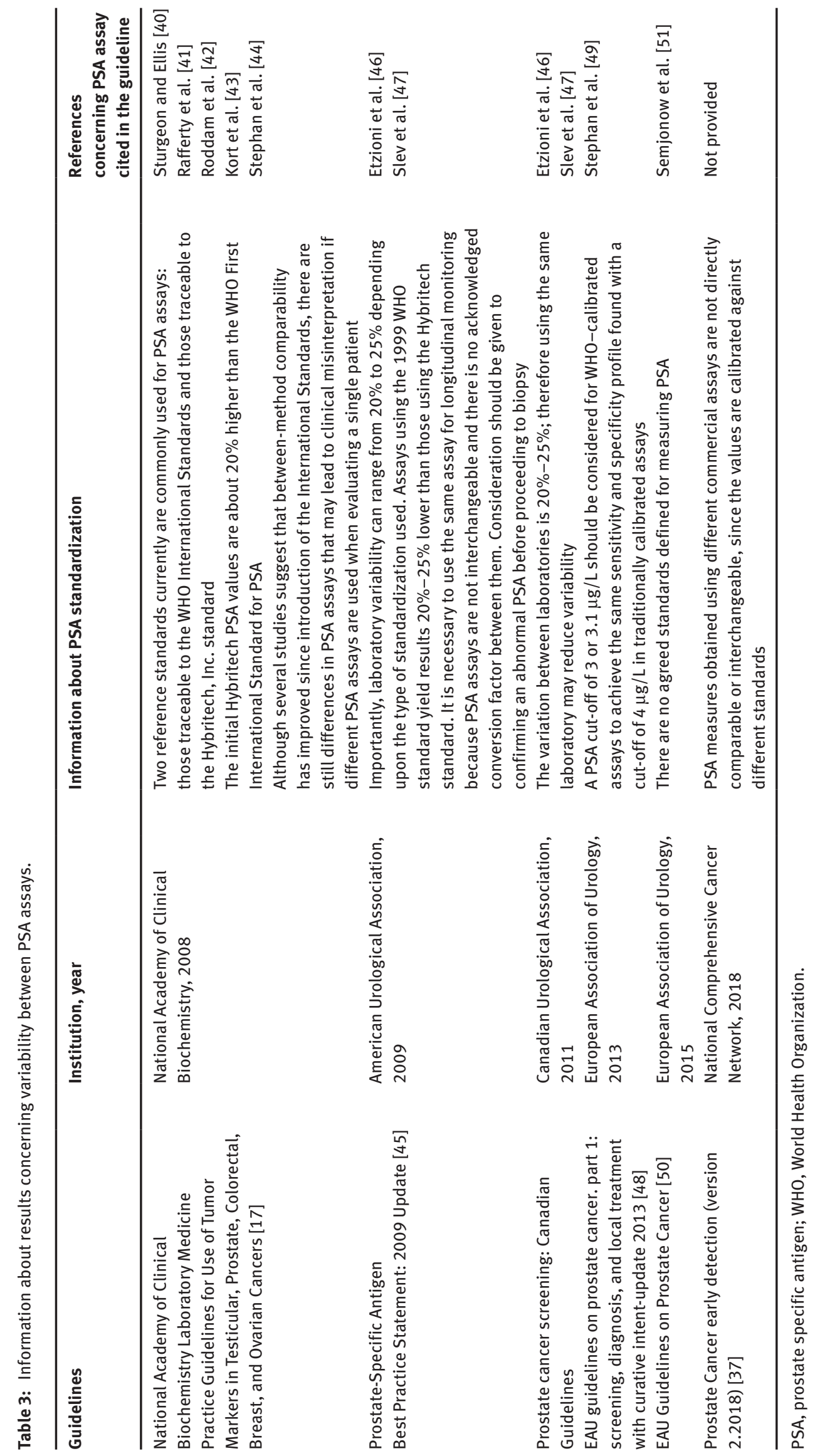




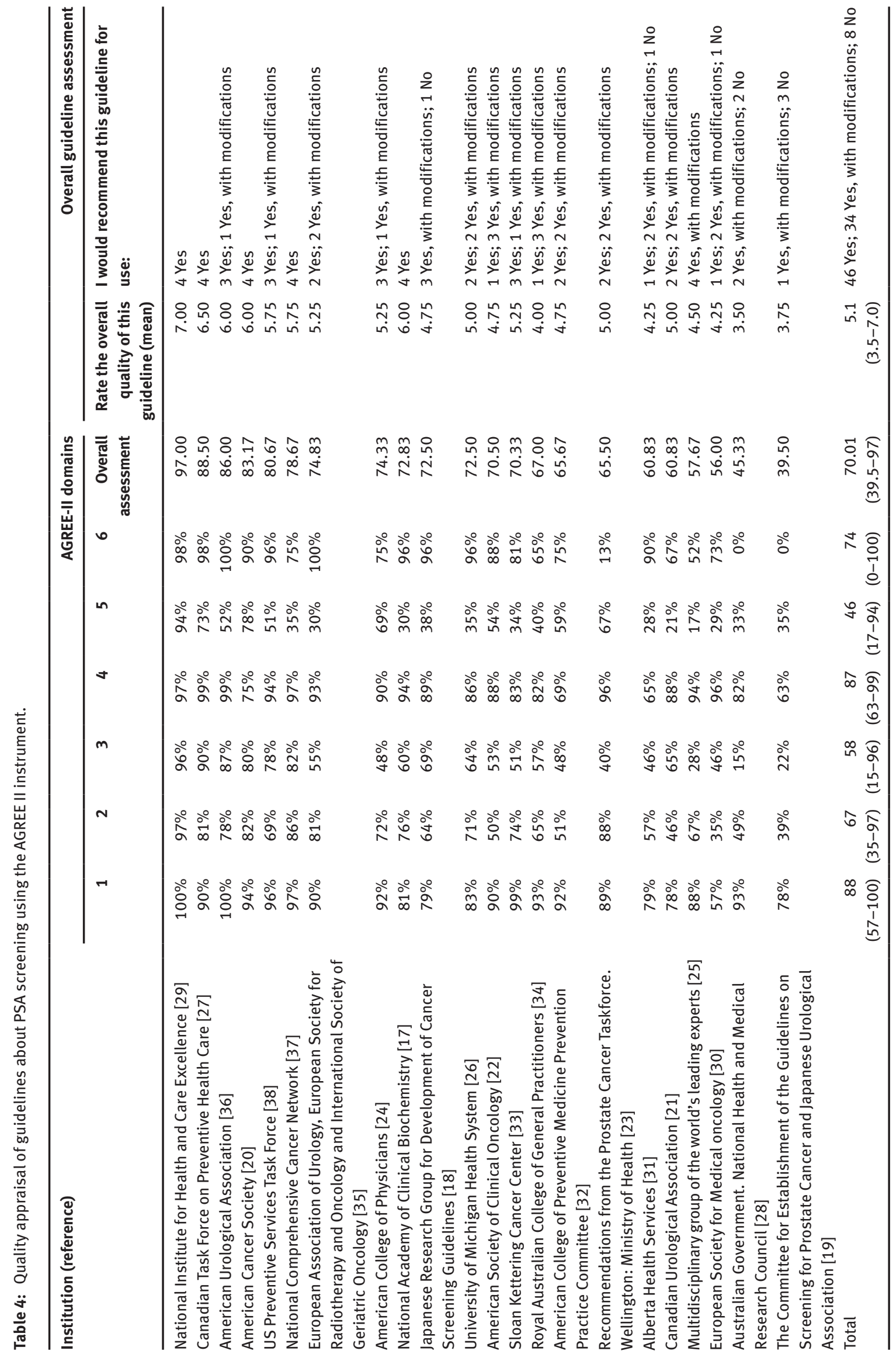


In our study, we found that recommendations were graded according to the level of evidence only in 12 of 22 guidelines evaluated and clear algorithms were only provided for four CPGs. On the other hand, we reviewed the sources of information used in every guideline evaluated in this study. It is remarkable that only one of 22 guidelines was not based on a wide review of data (Table 1). This procedure was specifically discarded by Vickers et al. [33], focusing their attention on three well-known screening high quality studies (ERSPC, the Prostate Cancer Prevention Trial and the Malmö Project Study), and arguing that regarding to other questions (frequency of PSA testing, indications for biopsy) a systematic review of the literature would be uninformative.

Finally, we found that laboratory issues are frequently $(86 \%)$ omitted in GPCs. In our opinion, the no inclusion of laboratory medicine specialists in the development process of the guidelines is the main reason for it. The laboratory medicine experts can provide the perspective of the clinical laboratory that sometimes goes unmarked among clinicians [12].

We found that laboratory medicine specialists participate in only two of the 22 CPGs reviewed in our study, one of them corresponding to the NACB guideline. This guideline widely considers factors in the pre-analytical, analytical and post-analytical phases, which can affect the clinical interpretation of PSA results. We suggest a list of laboratory issues for PSA assays (Table 2) that should be examined in the CPGs, following Aakre et al. [16] and the specific criteria for PSA reported by Schmeller [39].

The lack of harmonization of the assays is a major problem in the interpretation of PSA results. The establishment of the 1st International Standard for PSA (World Health Organization 96/670) in 2000 and its adoption as primary calibrator by most manufacturers of PSA assays decreases differences between assays, but concordance has not yet been achieved [49]. Additionally, the reference cut-off of $4 \mu \mathrm{g} / \mathrm{L}$ used to select patients for biopsy was obtained using the traditional Hybritech $($ standard. Information concerning the influence of WHO standard in the PSA cut-off was directly or indirectly reported by six guidelines (Table 3). Furthermore, the NACB, the 2009 AUA and the 2013 EAU guidelines explain that the initial Hybritech $\subset$ PSA values are about 20\% higher than the assays using the WHO 96/670 standard. The information furnished by the Canadian Urological Association guidelines is less precise, only reporting a $20 \%-25 \%$ variation among laboratories. In consequence, it would be necessary to change the cut-off of $4 \mu \mathrm{g} / \mathrm{L}$ for a cut-off of $3.1 \mu \mathrm{g} / \mathrm{L}$ to obtain similar clinical results. However, published data show that this is not absolutely true. Initial results comparing Access $(\subset)$ PSA test using WHO and Hybritech $\odot$ standards showed that PSA serum levels were 20\%-25\% lower when WHO standard is adopted [62]. Similar results have been observed for other assays using the reference material from WHO, but there are assays were the differences with the Hybritech(C) PSA assay are minimal despite the fact that the WHO standard is used. This point was remarked by Stephan et al. [44] showing that PSA serum levels measured with Elecsys $(\mathcal{C}$ (using the WHO standard) were very similar to the Hybritech $($ C PSA values. More recently, Foj et al. [63] compared different assays calibrated against the WHO standard in relation to the Hybritech $($ PSA values. The authors showed lower PSA results when Advia Centaur $(\subset$, and Architect $\subset$ were used, but results for Elecsys $\subset$, Lumipulse G $1200 \subset$, and Immulite 2000(C) were substantially similar to the Hybritech( PSA values.

Despite the availability of numerous CPGs considering PCa screening, consensus is currently lacking among them, although opportunistic screening is not recommended. Discrepant methodologies have been applied in the development of these CPGs, even in one case a systematic review of the literature has been explicitly rejected. Also, differences in the quality of the CPGs available have been reported by several authors. We focused our attention on the laboratory issues, because they are frequently omitted in the development of GPCs. The mechanical transfer of the established Hybritech $($ reference range of $<4.0 \mu \mathrm{g} / \mathrm{L}$ to other assays could lead to clinical mistakes in the selection of patients for biopsy. Differences between assays in the measurement of PSA serum levels are not taken into account by the majority of these guidelines. Furthermore, we detected some errors in three of the CPGs about the consequences of the calibration of PSA assays against the WHO. In conclusion, in our opinion, the quality of PCa early detection guidelines could be adequately improved considering the laboratory issues in their development.

Author contributions: All the authors have accepted responsibility for the entire content of this submitted manuscript and approved submission.

Research funding: None declared. Employment or leadership: None declared. Honorarium: None declared.

Competing interests: The funding organization(s) played no role in the study design; in the collection, analysis, and interpretation of data; in the writing of the report; or in the decision to submit the report for publication. 


\section{References}

1. Ferlay J, Colombet M, Soerjomataram I, Dyba T, Randi G, Bettio $M$, et al. Cancer incidence and mortality patterns in Europe: estimates for 40 countries and 25 major cancers in 2018. Eur J Cancer 2018;103:356-87.

2. Schröder FH, Hugosson J, Roobol MJ, Tammela TL, Ciatto S, Nelen V, et al. Screening and prostate-cancer mortality in a randomized European study. N Engl J Med 2009;360:1320-8.

3. Andriole GL, Crawford ED, Grubb RL, Buys SS, Chia D, Church TR, et al. Mortality results from a randomized prostate-cancer screening trial. N Engl J Med 2009;360:1310-9.

4. Schröder FH, Hugosson J, Roobol MJ, Tammela TL, Zappa $M$, Nelen V, et al. Screening and prostate cancer mortality: results of the European Randomized Study of Screening for Prostate Cancer (ERSPC) at 13 years of follow-up. Lancet 2014;384:2027-35.

5. Chou R, Croswell JM, Dana T, Bougatsos C, Blazina I, Fu R, et al. Screening for prostate cancer: a review of the evidence for the U.S. Preventive Services Task Force. Ann Intern Med 2011;155:762-71.

6. Schroeder FH, Hugosson J, Carlsson S, Tammela T, Määttänen L, Auvinen A, et al. Screening for prostate cancer decreases the risk of developing metastatic disease: findings from the European Randomized Study of Screening for Prostate Cancer (ERSPC). Eur Urol 2012;62:745-52.

7. Buzzoni C, Auvinen A, Roobol MJ, Carlsson S, Moss SM, Puliti $D$, et al. Metastatic prostate cancer incidence and prostatespecific antigen testing: new insights from the European Randomized Study of Screening for Prostate Cancer. Eur Urol 2015;68:885-90.

8. Gulati R, Tsodikov A, Etzioni R, Hunter-Merrill RA, Gore JL, Mariotto $A B$, et al. Expected population impacts of discontinued prostate-specific antigen screening. Cancer 2014;120:3519-26.

9. Stephan C, Rittenhouse H, Hu X, Cammann H, Jung K. ProstateSpecific Antigen (PSA) screening and new biomarkers for prostate cancer (PCa). EJIFCC 2014;25:55-78.

10. Carlsson SV, Roobol MJ. What's new in screening in 2015? Curr Opin Urol 2016;26:447-58.

11. Eapen RS, Herlemann A, Washington 3rd SL, Cooperberg MR. Impact of the United States Preventive Services Task Force 'D' recommendation on prostate cancer screening and staging. Curr Opin Urol 2017;27:205-9.

12. Giménez N, Filella X, Gavagnach M, Allué JA, Pedrazas D, Ferrer $F$, et al. [Prostate cancer screening using prostate-specific antigen: The views of general and laboratory physicians]. Semergen 2018;44:409-19.

13. National Guidelines Clearinghouse. Available at: https://www. guideline.gov/help-and-about/search-browse/search-tips. Accessed: 5 Oct 2018.

14. Guidelines International Network Web site. Available at: https:// www.g-i-n.net/. Accessed: 5 Oct 2018.

15. NICE. National Institute for Health and Care Excellence Web site. Available at: https://www.nice.org.uk/. Accessed: 5 Oct 2018.

16. Aakre KM, Langlois MR, Watine J, Barth JH, Baum H, Collinson $P$, et al. Critical review of laboratory investigations in clinical practice guidelines: proposals for the description of investigation. Clin Chem Lab Med 2013;51:1217-26.
17. Sturgeon CM, Duffy MJ, Stenman UH, Lilja H, Brünner N, Chan DW, et al. National Academy of Clinical Biochemistry laboratory medicine practice guidelines for use of tumor markers in testicular, prostate, colorectal, breast, and ovarian cancers. Clin Chem 2008;54:e11-79.

18. Hamashima C, Nakayama T, Sagawa M, Saito H, Sobue T. The Japanese guideline for PCa screening. Jpn J Clin Oncol 2009;39:339-51.

19. Updated Japanese Urological Association Guidelines on prostate-specific antigen-based screening for prostate cancer in 2010. The Committee for Establishment of the Guidelines on Screening for Prostate Cancer and Japanese Urological Association. Int J Urol 2010;17:830-8.

20. Wolf AM, Wender RC, Etzioni RB, Thompson IM, D'Amico AV, Volk RJ, et al. American Cancer Society PCa Advisory Committee. CA Cancer J Clin 2010;60:70-98.

21. Izawa JI, Klotz L, Siemens DR, Kassouf W, So A, Jordan J, et al. Prostate cancer screening: Canadian guidelines 2011. Can Urol Assoc J 2011;5:235-40.

22. Basch E, Oliver TK, Vickers A, Thompson I, Kantoff P, Parnes H, et al. Screening for prostate cancer with prostate-specific antigen testing: American Society of Clinical Oncology Provisional Clinical Opinion. J Clin Oncol 2012;30:3020-5.

23. Prostate Cancer Taskforce. Diagnosis and management of prostate cancer in New Zealand men: recommendations from the Prostate Cancer Taskforce. Wellington: Ministry of Health, 2012. Available at: http://www.prostate.org.nz/documents/diagnosismanagement-prostate-cancer-nz-men_(3).pdf. Accessed: 9 oct 2018.

24. Qaseem A, Barry MJ, Denberg TD, Owens DK, Shekelle P. Screening for PCa: a guidance statement from the Clinical Guidelines Committee of the American College of Physicians. Ann Intern Med 2013;158:761-9.

25. Murphy DG, Ahlering T, Catalona WJ, Crowe H, Crowe J, Clarke N, et al. The Melbourne Consensus Statement on the early detection of PCa. BJU Int 2014;113:186-8.

26. Cancer Screening. Guidelines for clinical care ambulatory. University of Michigan, 2014. Available at: https://www.med.umich. edu/1info/FHP/practiceguides/adult.cancer/cancergline.pdf. Accessed: 9 Oct 2018

27. Recommendations on screening for prostate cancer with the prostate-specific antigen test. Canadian task force on preventive health care. Can Med Assoc J 2014;186:1225-34.

28. PSA Testing for Prostate Cancer in Asymptomatic Men Information for Health Practitioners. Australian Government. National Health and Medical Research Council, 2014. Available at: https://nhmrc.gov.au/sites/default/files/documents/reports/ clinical\%20guidelines/men4d-psa-testing-asymptomatic.pdf. Accessed: 9 Oct 2018.

29. National Collaborating Centre for Cancer. Prostate cancer: diagnosis and treatment. London (UK): National Institute for Health and Care Excellence (NICE), 2014. Available at: https:// www.nice.org.uk/guidance/cg175/evidence/full-guideline-191710765. Accessed: 9 Oct 2018.

30. Parker C, Gillessen S, Heidenreich A, Horwich A, on behalf of the ESMO Guidelines Committee. Prostate cancer: ESMO Clinical Practice Guidelines for diagnosis, treatment and follow-up. Ann Oncol 2015;26(Supplement 5):v69-77.

31. Alberta Health Services. Prostate Cancer. Clinical practice guideline Alberta, 2015. 
32. Livingston C), Freeman RJ, Mohammad A, Costales VC, Titus TM, Harvey BJ, et al. Choosing Wisely ${ }^{\circledR}$ in preventive medicine: the American College of Preventive Medicine's Top 5 List of Recommendations. Am J Prev Med 2016;51:141-9.

33. Vickers AJ, Eastham JA, Scardino PT, Lilja H. The Memorial Sloan Kettering Cancer Center recommendations for PCa screening. Urology 2016;91:12-8.

34. The Royal Australian College of General Practitioners. Guidelines for preventive activities in general practice. 9th edn. East Melbourne, Vic: RACGP, 2016. Available at: https://www.racgp. org.au/your-practice/guidelines/redbook/9-early-detection-ofcancers/91-prostate-cancer/. Accessed: 9 Oct 2018.

35. Mottet N, Bellmunt J, Bolla M, Briers E, Cumberbatch MG, De Santis M, et al. EAU-ESTRO-SIOG guidelines on prostate cancer. Part 1: screening, diagnosis, and local treatment with curative intent. Eur Urol 2017;71:618-29.

36. Carter HB, Albertsen PC, Barry MJ, Etzioni R, Freedland SJ, Greeneet KL, et al. Early detection of prostate cancer. American Urological Association, 2018. Available at: https://www.auanet. org/guidelines/prostate-cancer-early-detection-guideline. Accessed: 19 March 2019.

37. NCCN Clinical Practice Guidelines in Oncology. Prostate Cancer Early Detection. Version 2.2018, April 2018. Available at: https:// www.nccn.org/store/login/login.aspx?ReturnURL=https://www. nccn.org/professionals/physician_gls/pdf/prostate_detection. pdf. Accessed: 9 Oct 2018.

38. Grossman DC, Curry SJ, Owens DK, Bibbins-Domingo K, Caughey $A B$, Davidson KW, et al. Screening for prostate cancer: US Preventive Services Task Force recommendation statement. J Am Med Assoc 2018;319:1901-13.

39. Schmeller N. Clinical value of PSA. Bremen: UNI-Med Verlag, 2005.

40. Sturgeon CM, Ellis AR. Improving the comparability of immunoassays for prostate-specific antigen (PSA): progress and problems. Clin Chim Acta 2007;381:8-92.

41. Rafferty B, Rigsby P, Rose M, Stamey T, Gaines DR. Reference reagents for prostate-specific antigen (PSA): establishment of the first international standards for free PSA and PSA (90:10). Clin Chem 2000;46:1310-7.

42. Roddam AW, Rimmer J, Nickerson C, Ward AM. Prostate-specific antigen: bias and molarity of commercial assays for PSA in use in England. Ann Clin Biochem 2006;43:35-48.

43. Kort SA, Martens F, Vanpoucke H, van Duijnhoven HL, Blankenstein MA. Comparison of 6 automated assays for total and free prostate specific antigen with special reference to their reactivity toward the WHO 96/670 reference preparation. Clin Chem 2006;52:1568-74.

44. Stephan C, Klaas M, Muller C, Schnorr D, Loening SA, Jung K. Interchangeability of measurements of total and free prostate specific antigen in serum with 5 frequently used assay combinations: an update. Clin Chem 2006;52:59-64.

45. Greene KL, Albertsen PC, Babaian RJ, Carter HB, Gann PH, Han $M$, et al. Prostate specific antigen best practice statement: 2009 update. J Urol 2009;182:2232-41.

46. Etzioni RD, Ankerst DP, Weiss NS, Inoue LY, Thompson IM. Is prostate-specific antigen velocity useful in early detection of prostate cancer? A critical appraisal of the evidence. J Natl Cancer Inst 2007;99:1510-5.
47. Slev PR, La'ulu SL, Roberts WL. Intermethod differences in results for total PSA, free PSA, and percentage of free PSA. Am J Clin Pathol 2008;129:952-8.

48. Heidenreich A, Bastian PJ, Bellmunt J, Bolla M, Joniau S, van der Kwast T, et al. EAU guidelines on prostate cancer. Part 1: screening, diagnosis, and local treatment with curative intent-update 2013. Eur Urol 2014;65:124-37.

49. Stephan C, Köpke T, Semjonow A, Lein M, Deger S, Schrader M, et al. Discordant total and free prostate-specific antigen (PSA) assays: does calibration with WHO reference materials diminish the problem? Clin Chem Lab Med 2009;47:1325-31.

50. Mottet N, Bellmunt J, Briers E, van den Bergh RC, Bolla M, van Casteren NJ, et al. EAU Guidelines on prostate cancer. Available at: https://uroweb.org/wp-content/uploads/09-Prostate-Cancer_LR.pdf. Accessed: 10 Oct 2018.

51. Semjonow A, Brandt B, Oberpenning F, Roth S, Hertle L. Discordance of assay methods creates pitfalls for the interpretation of prostate specific antigen values. Prostate Suppl 1996;7:3-16.

52. Moyer VA, U.S. Preventive Services Task Force. Screening for PCa: U.S. Preventive Services Task Force recommendation statement. Ann Intern Med 2012;157:120-34.

53. Aslani A, Minnillo BJ, Johnson B, Cherullo EE, Ponsky LE, Abouassaly $R$. The impact of recent screening recommendations on prostate cancer screening in a large health care system. J Urol 2014;191:1737-42.

54. Cohn JA, Wang CE, Lakeman JC, Silverstein JC, Brendler CB, Novakovic KR, et al. Primary care physician PSA screening practices before and after the final U.S. Preventive Services Task Force recommendation. Urol Oncol 2014;32:41.e23-30.

55. Ong MS, MandI KD. Trends in Prostate-Specific Antigen screening and prostate cancer interventions 3 years after the U.S. Preventive Services Task Force recommendation. Ann Intern Med 2017;166:451-2.

56. Fleshner K, Carlsson SV, Roobol MJ. The effect of the USPSTF PSA screening recommendation on prostate cancer incidence patterns in the USA. Nat Rev Urol 2017;14:26-37.

57. Calonge N, Petitti DB, DeWitt TG, Dietrich AJ, Gregory KD, Harris $R$, et al. Screening for prostate cancer: U.S. Preventive Services Task Force recommendation statement. Ann Intern Med 2008;149:185-91.

58. Heijnsdijk EA, Denham D, de Koning HJ. The cost-effectiveness of prostate cancer detection with the use of Prostate Health Index. Value Health 2016;19:153-7.

59. Mathieu R, Castelli C, Fardoun T, Peyronnet B, Shariat SF, Bensalah $\mathrm{K}$, et al. Cost analysis of prostate cancer detection including the prostate health index (PHI). World J Urol 2019;37:481-7.

60. Palenius E, Bonnevier E. Evaluation of the cost-effectiveness of novel tests in the screening and diagnostic. Phases of prostate cancer compared to current practice. Lund University, 2017.

61. Gupta M, McCauley J, Farkas A, Gudeloglu A, Neuberger MM, Ho YY, et al. Clinical practice guidelines on prostate cancer: a critical appraisal. J Urol 2015;193:1153-8.

62. Stephan C. WHO standardization of PSA tests: clinical consequences. Nat Rev Urol 2009;6:303-5.

63. Foj L, Filella X, Alcover J, Augé JM, Escudero JM, Molina R. Variability of assay methods for total and free PSA after WHO standardization. Tumor Biol 2014;35:1867-73. 